\title{
REMARKS ON LINEAR DIFFERENTIAL SYSTEMS WITH MEASURABLE COEFFICIENTS
}

\author{
RUSSELL A. JOHNSON
}

\begin{abstract}
We discuss several topics having to do with linear differential equations with locally integrable coefficients. These include gap labelling and Cantor spectrum for the random Schrodinger operator, and Coppel's perturbation theorem.
\end{abstract}

1. Introduction. The purpose of this paper is to discuss several matters considered in recent papers dealing with the theory of ordinary linear differential equations

$$
x^{\prime}=y(t) x \quad\left(x \in \mathbf{C}^{n}, t \in \mathbf{R}\right)
$$

and with the spectral theory of ordinary linear differential operators. The topics we treat are rather diverse, including among others gap labelling for random differential and difference operators, a theorem of Coppel [6] concerning roughness of exponential dichotomy, and Cantor spectrum for the almost periodic Schrodinger operator. Nevertheless, the methods we use provide a unifying theme: we regard $y$ as just one element of a compact, translation invariant set $Y$ of coefficient matrices, and apply techniques of topological dynamics and dynamical systems to the corresponding family of ODEs (1). Thus this paper forms part of a now substantial body of work (e.g., $[\mathbf{1 5}, \mathbf{1 9}, \mathbf{3 1}, \mathbf{3 2}]$; see $[\mathbf{3 5}]$ for fundamental facts and reference to earlier literature).

Three sections follow this introduction. $\S 2$ is devoted to the "random" differential equation (1); i.e., the coefficient $y(t)=y_{t}$ is a stochastic process with values in the set $\operatorname{gl}(n, \mathbf{C})$ of complex $n \times n$ matrices. We first show how many such equations can be put into a context in which tools of topological dynamics apply. The material is not new and is elementary but it is hoped that its presentation here will be of some use, to someone. In any case, we use it to provide a general framework in which results of several recent papers are valid $[\mathbf{7}, \mathbf{1 5}, \mathbf{1 6}, \mathbf{1 9}]$. These results are often stated under unnecessarily restrictive hypotheses, as we indicate. They include gap labelling; in particular we "explain" a result of Bellissard and Scoppola [3] from our topological point of view. We also discuss the relation between spectrum and exponential dichotomy for linear differential operators [15], and a characterization due to De Concini and Johnson [7] of the algebro-geometric potentials for the Schrodinger [27, 23] and AKNS [1] operators.

In $\S 3$ we consider Coppel's perturbation theorem [6]. To state it, recall that (1) is said to have exponential dichotomy if there is a projection $P: \mathbf{C}^{n} \rightarrow \mathbf{C}^{n}$ and constants $K>0, \alpha>0$ such that, if $\Phi(t)$ is the fundamental matrix solution of

Received by the editors April 14, 1986.

1980 Mathematics Subject Classification (1985 Revision). Primary 34C35, 54H20.

Key words and phrases. Exponential dichotomy, Cantor spectrum. 
(1) with $\Phi(0)=I$, then

$$
\begin{gathered}
\left\|\Phi(t) P \Phi^{-1}(s)\right\| \leq K e^{-\alpha(t-s)} \quad(t \geq s), \\
\left\|\Phi(t)(I-P) \Phi^{-1}(s)\right\| \leq K e^{\alpha(t-s)} \quad(t \leq s) .
\end{gathered}
$$

Coppel shows that, if $y(t)$ is continuous, and if $v(t)$ is continuous with $\|v\|_{\infty}=$ $\sup _{t}\|v(t)\| \leq \delta$, then for sufficiently small $\delta$, the perturbed system

$$
x^{\prime}=(y(t)+v(t)) x
$$

also has exponential dichotomy with, say, projection $P(v)$, and moreover $\|P(v)-P\| \leq K\|v\|_{\infty}$ where $K$ depends only on equation (1).

Our result is a partial generalization of Coppels in the sense that $y(t)$ is replaced by a compact, translation-invariant set $Y$ of measurable coefficient matrices, and the perturbation $v$ also lies in such a set. In addition, our proof differs substantially from Coppel's; in particular, we use ideas of Hirsch, Pugh, and Shub [14] and the spectral theorem for linear operators to prove the Lipschitz estimate on the projection $P(v)$. The Lipschitz continuity in $v$ is especially important, since it can often be "bootstrapped" into actual differentiable dependence of $P$ on $v$ (see, e.g., Palmer [29] or [16] for situations in which this is true).

Sacker and Sell [32] have also proven a roughness result which, however, does not seem to lend itself to a Lipschitz continuity statement. On the other hand, the formulation of our theorem is influenced by the Sacker-Sell statement. Moreover, part of our proof relies on arguments used by them in the proof of their theorem.

Finally, in $\S 4$, we consider the one-dimensional Schrodinger operator

$$
L=-d^{2} / d t^{2}+q(t)
$$

with (Bohr) almost periodic potential $q(t)$. It is conjectured that, for generic $q$, the spectrum $\Sigma$ of $L$ is a Cantor set, i.e., if $L$ is viewed as a selfadjoint operator on $L^{2}(\mathbf{R})$, then $\{\lambda \in \mathbf{C} \mid \lambda-L$ is not invertible $\}$ is a closed nowhere dense subset of $\mathbf{R}$.

We prove a result which supports this conjecture. Namely, given some reasonable Banach space $B$ of almost periodic potentials $q$, we find a residual subset $B_{1} \subset B$ such that, if $q \in B_{1}$, then $L=L_{q}$ has at least one of the following properties: (i) $\Sigma=\Sigma_{q}$ is a Cantor set; or (ii) if $I \subset \Sigma$ is a nondegenerate interval, then the Lyapounov exponent (see $\S 4) \beta=\beta(\lambda)$ of $L_{q} \phi=\lambda \phi$ is strictly positive on a subset $I_{1} \subset I$ of positive Lebesgue measure. Since $\beta(\lambda)$ measures exponential growth of solutions $\phi$ of $L_{q} \phi=\lambda \phi$, one expects that (ii) implies (i); i.e., for a generic perturbation of $q^{\prime}$ of $q, L_{q^{\prime}}$ has Cantor spectrum. However we have not been able to prove this.

It should be emphasized that our proof uses neither smoothness of $q$ nor Diophantine conditions on rotation numbers. Instead, we use results of Johnson and Moser [18], Moser and Poschl [26], and De Concini and Johnson [7], which are valid independently of such assumptions.

2. Random differential equations. We consider the differential equations

$$
x^{\prime}=Z_{t}(\omega) x \quad\left(x \in \mathbf{C}^{n}, \omega \in \Omega\right),
$$

where $\Omega$ is a measure space with probability measure $\mu$, and for each $t \in \mathbf{R}, \Omega \rightarrow$ $\operatorname{gl}(n, \mathbf{C})$ is a $\mu$-measurable function. We will give conditions on the "random process" $\left\{Z_{t} \mid t \in \mathbf{R}\right\}$ under which methods of topological dynamics may be used to 
study equations $(2)_{\omega}$; in particular, we are interested in conditions assuring that the solutions generate a continuous cocycle (see 2.3).

We make the following assumptions on $\left\{Z_{t} \mid t \in \mathbf{R}\right\}$.

(i) (Stochastic continuity) Given $\varepsilon>0$, there exists $\delta>0$ such that, if $|h|<\delta$, then $\mu\left\{\omega \mid\left\|Z_{t+h}(\omega)-Z_{t}(\omega)\right\|>\varepsilon\right\}<\varepsilon$. Here and below $\|\cdot\|$ is some fixed norm on $\operatorname{gl}(n, \mathbf{C})$. It follows [9] that we can modify each $Z_{t}$ on a set of $\mu$-measure zero in such a way that the map $(t, \omega) \rightarrow Z_{t}(\omega)$ is measurable with respect to the product of Lebesgue measure $m$ on $\mathbf{R}$ with $\mu$.

(ii) (Stationarity) If $t_{1}, \ldots, t_{k} \in \mathbf{R}$ and $A_{1}, \cdots, A_{k}$ are Borel subsets of $\mathrm{gl}(n, \mathbf{C})$, then the joint distribution $\mu\left\{\omega \mid Z_{t_{1}}(\omega) \in A_{1}, \ldots, Z_{t_{k}}(\omega) \in A_{k}\right\}$ depends only on the differences $t_{2}-t_{1}, \ldots, t_{k}-t_{k-1}$.

Let us now embed the functions $t \rightarrow Z_{t}(\omega)$ in a compact subset $Y$ of a function space $E$ in such a way that $\mu$ defines a Radon probability measure on $Y$. There are many ways of doing this; we single out the following, which has proved to be useful.

For $1 \leq p<\infty$, let $E_{p}=\left\{\eta: \mathbf{R} \rightarrow \operatorname{gl}(n, \mathbf{C}) \mid \eta\right.$ is measurable and $\left.\|\eta\|_{p}<\infty\right\}$, where

$$
\|\eta\|_{p}^{p}=\sup _{t} \int_{t}^{t+1}\|\eta(s)\|^{p} d s
$$

if $1 \leq p<\infty$, and $\|\eta\|_{\infty}=\sup _{t}\|\eta(t)\|$. Thus $E_{p}$ is a vector subspace of $L_{\text {loc }}^{p}(\mathbf{R}, \operatorname{gl}(n, \mathbf{C}))$. Let $\eta^{i j}(\cdot)$ be the $i j$ th component of the matrix function $\eta(\cdot)(1 \leq$ $i, j \leq n)$. Give $E_{p}$ the weak topology defined as follows. Let $q$ satisfy $1 / p+1 / q=1$, and let $\phi \in L^{q}(\mathbf{R})$ have compact support; we say that $\eta_{r} \rightarrow \eta$ iff for each such $\phi$,

$$
\int_{-\infty}^{\infty} \eta_{r}^{i j}(s) \phi(s) d s \rightarrow \int_{-\infty}^{\infty} \eta^{i j}(s) \phi(s) d s .
$$

Next suppose that, for $\mu$-a.a. $\omega$, there exists a constant $M$ independent of $\omega$ such that

$$
\sup _{t} \int_{t}^{t+1}\left\|Z_{s}(\omega)\right\|^{p} d s \leq M
$$

If $p=1$, suppose in addition that

$$
\lim _{\varepsilon \rightarrow 0^{+}} \sup _{t} \int_{t}^{t+\varepsilon}\left\|Z_{s}(\omega)\right\| d s=0
$$

for $\mu$-a.a. $\omega$. Replacing $\Omega$ by a subset of $\mu$-measure 1 if necessary, we assume that these conditions hold for all $\omega \in \Omega$.

Define $k: \Omega \rightarrow E_{p}: k(\omega)(t)=Z_{t}(\omega)$. Let $Y$ be the closure of $k(\Omega) \subset E_{p}$ in the weak topology. Assume condition $(3)_{p}$ (and condition (4) if $p=1$ ). Then $Y$ is compact and metrizable.

Let us check that $k: \Omega \rightarrow Y$ is Borel measurable; i.e., if $B \subset Y$ is a Borel set, then $k^{-1}(B)$ is $\mu$-measurable. In fact, let $\phi \in L^{q}(\mathbf{R})$ have compact support. Fix $1 \leq i, j \leq n$, and

$$
f_{\phi}^{i j}(y)=\int_{\mathbf{R}} y^{i j}(s) \phi(s) d s \quad(y \in Y) .
$$

Then $f_{\phi}^{i j} \circ k$ is $\mu$-measurable because $Z_{t}(\omega)$ is jointly measurable. The subalgebra of $C(Y)$ generated by $\left\{f_{\phi}^{i j}\right\}$ separates points in $Y$, hence generates $C(Y)$. Hence 
$f \circ k$ is $\mu$-measurable for all $f \in C(Y)$, and Borel measurability of $k$ follows from metrizability of $Y$.

Now define a bounded linear functional $\hat{\mu}$ on $C(Y)$ by

$$
\hat{\mu}(f)=\int_{\Omega}(f \circ k)(\omega) d \mu(\omega) .
$$

Then $\hat{\mu}(f) \geq 0$ when $f \geq 0$, and $\hat{\mu}(1)=1$. Let $\nu$ be an associated Radon probability measure. One shows that $\mu\left(k^{-1}(B)\right)=\nu(B)$ for each Borel $B \subset Y$. Thus $k$ defines a measure-homomorphism from $(\Omega, \mu)$ to $(Y, \nu)$ (with the Borel field on $Y$ ).

In our construction of $(Y, \nu)$, we have used only stochastic continuity and assumptions $(3)_{p},(4)$. We now consider stationarity: this of course translates into invariance of $\nu$ under the translation flow $\tau$ on $Y$. Here $\tau: Y \times \mathbf{R} \rightarrow Y$ is defined by

$$
\tau(y, t)(s)=y(t+s) \quad(y \in Y ; t, s \in \mathbf{R}) .
$$

The map $\tau$ is jointly continuous. We write $\tau_{t}(y)=\tau(y, t)$. The measure $\nu$ is said to be invariant if $\nu\left(\tau_{t}(B)\right)=\nu(B)$ for each Borel $B \subset Y$.

Using stationarity and simple arguments which we omit, one shows

2.1. Statement. The measure $\nu$ constructed above is invariant.

Ergodicity of $\left\{Z_{t}\right\}$ also carries over to $(Y, \nu)$. We refer to Doob [9, pp. $464 \mathrm{ff}$.] for the definition and a discussion of this concept. We only note that, applying his constructions to a stochastically continuous and stationary process $\left\{Z_{t}\right\}$, one obtains a one-parameter group of unitary transformations $U_{t}: L^{2}(\Omega, \mu) \rightarrow L^{2}(\Omega, \mu)$. One says that $\left\{Z_{t}\right\}$ is ergodic if the only functions $f$ satisfying $U_{t} f=f$ for all $t \in \mathbf{R}$ are the constant functions. Now, if $g \in L^{2}(Y, \nu)$ satisfies $g \circ \tau_{t}=g \nu$-a.e. for each $t \in \mathbf{R}$, then one can show that $f=g \circ k$ satisfies $U_{t} f=f(t \in \mathbf{R})$. Hence $g=$ const $\nu$-a.e. Using a standard argument, one has

2.2. Statement. If $\left\{Z_{t}\right\}$ is ergodic (thus in particular stationary), then $\nu$ is ergodic; i.e., $\nu$ is invariant, and, if $B \subset Y$ is a Borel set such that $\nu\left(B \triangle \tau_{t} B\right)=0$ for each $t \in \mathbf{R}(\triangle=$ symmetric difference), then $\nu(B)=0$ or $\nu(B)=1$.

Our attitude now is that the random differential equations $(2)_{\omega}$ have been translated into the family of ODEs

$$
x^{\prime}=y(t) x \quad\left(x \in \mathbf{C}^{n}, y \in Y\right) .
$$

We can apply techniques of topological dynamics to study equations $(1)_{y}$ because of the following easy result, whose proof is omitted.

2.3. LEMMA. Let $Y \subset E_{p}$ be weakly compact and invariant under $\tau$. Let $\Phi_{y}(t)$ be the fundamental matrix solution of $(1)_{y}$ satisfying $\Phi_{y}(0)=I$. Then $\Phi_{\tau_{t}(y)}(s) \circ$ $\Phi_{y}(t)=\Phi_{y}(t+s)(y \in Y ; t, s \in \mathbf{R})$, and $\Phi: Y \times \mathbf{R} \rightarrow \mathrm{GL}(n, \mathbf{C})$ is jointly continuous. That is, $\Phi$ defines a continuous cocycle on $(Y, \tau)[\mathbf{1 0}, 11]$.

Before considering applications of the construction of $(Y, \nu)$, we make a few observations.

2.4. REMARKS. (a) Let $Y$ be a subset of $E_{p}(p>1)$ for which there exists a constant $M$ such that

$$
\sup _{t} \int_{t}^{t+1}\|y(s)\|^{p} d s \leq M \quad(y \in Y) .
$$

Then the distribution topology on $Y$ agrees with the weak topology. 
(b) In [21], Kotani considers the one-dimensional Schrodinger equation

$$
\left(\frac{-d^{2}}{d t^{2}}+y(t)\right) \phi=\lambda \phi
$$

with $y \in H=L^{2}\left(\mathbf{R},\left(1+|t|^{3}\right)^{-1} d t\right)$. If $Y$ is a weakly compact and translation invariant subset of $H$, then $Y$ defines a weakly compact and translation invariant subset of $E_{2}$. Thus our framework includes Kotani's if we insist on (weak) compactness of the coefficient space $Y$.

(c) One might prefer to do without condition (4) if $p=1$. The basic problem if one omits (4) is the following: The weak closure of a translation-invariant set satisfying (3) $)_{1}$ need not be weakly compact in $E_{1}$. If we enlarge $E_{1}$ to include measure-valued maps $y$, then we obtain a compact $Y$, but the cocycle $\Phi_{y}(t)$ need not be continuous in $t$.

Let us now briefly consider how certain results of $[\mathbf{7}, \mathbf{1 5}, \mathbf{1 6}, \mathbf{1 9}]$ can be applied to random ordinary differential equations of type $(2)_{\omega}$.

2.5. Spectrum and exponential dichotomy. Consider the one-dimensional, random Schrodinger equation

$$
L_{\omega} \phi=\left(\frac{-d^{2}}{d t^{2}}+Z_{t}(\omega)\right) \phi=\lambda \phi,
$$

or in system form

$$
x^{\prime}=\left(\begin{array}{c}
\phi \\
\phi^{\prime}
\end{array}\right)=\left(\begin{array}{cc}
0 & 1 \\
-\lambda+Z_{t}(\omega) & 0
\end{array}\right) x
$$

Assume that

$$
\omega \rightarrow\left(\begin{array}{cc}
0 & 1 \\
-\lambda+Z_{t}(\omega) & 0
\end{array}\right)
$$

satisfies (3) for some $p \geq 1$ (and (4) if $p=1$ ) for some (hence all) $\lambda \in \mathbf{C}$. We obtain differential equations

$$
x^{\prime}=\left(\begin{array}{cc}
0 & 1 \\
-\lambda+y(t) & 0
\end{array}\right) x \quad(y \in Y),
$$

where $Y$ is a weakly compact, translation-invariant subset of $L_{\mathrm{loc}}^{p}(\mathbf{R})$.

Using arguments of [15], we now obtain the following results.

(i) If $\operatorname{Im} \lambda \neq 0$, then equations (5) ${ }_{y}$ have exponential dichotomy. Thus each Schrodinger operator $L_{y}=-d^{2} / d t^{2}+y(t)$ is in the limit-point case at $t= \pm \infty$ ([5]; this follows from known limit-point criteria), and nonzero solutions $\phi_{ \pm}(t)$ of $L_{y} \phi=\lambda \phi$ which are in $L^{2}(0, \pm \infty)$ decay exponentially as $t \rightarrow \pm \infty$ at an exponential rate which is bounded away from zero independent of $y \in Y$.

(ii) If the orbit $\left\{\tau_{t}(y) \mid t \in \mathbf{R}\right\}$ is dense in $Y$, then $\left\{\lambda \in \mathbf{R} \mid\left(\lambda-L_{y}\right)^{-1}\right.$ is bounded in $\left.L^{2}(\mathbf{R})\right\} \equiv$ resolvent of $L_{y}$ equals $\{\lambda \in \mathbf{R} \mid$ equations (5) $y$ have ED $\}$. If $\nu$ is ergodic and $Y=\operatorname{Support}(\nu)$, then, for $\nu$-a.a. $y$, the orbit through $y$ is dense in $Y$. One can show that, in our construction of $(Y, \nu), k^{-1}$ (Support $\nu$ ) has $\mu$-measure 1 in $\Omega$ if $(\Omega, \mu)$ is ergodic. Thus for a stationary ergodic process $\left\{Z_{t}\right\}$ as considered here, almost all operators $L_{\omega}$ satisfy the hypothesis of (ii).

2.6. Gap labelling. One can use 2.5 to study "gap labelling," which refers to a systematic assignment of real numbers to intervals in the resolvent of $L_{\omega}$. Suppose 
that $\mu$ is ergodic, so that $\nu$ is also, and that $Y=\operatorname{Support}(\nu)$. Following Schwarzmann [33], introduce the group $\check{H}^{1}(Y ; \mathbf{Z})$ of equivalence classes of continuous maps $\phi: Y \rightarrow K=$ unit circle under the equivalence relation $\phi \sim \psi \Leftrightarrow \phi(y) \overline{\psi(y)}=$ $\exp i R(y)$ for a continuous function $R: Y \rightarrow \mathbf{R}$. The group operation on $\breve{H}^{1}(Y ; \mathbf{Z})$ is induced by pointwise multiplication of mappings. Define

$$
h: \check{H}^{1}(Y ; \mathbf{Z}) \rightarrow \mathbf{R}:[\phi] \rightarrow \lim _{t \rightarrow \infty} \frac{1}{t} \operatorname{Arg} \phi\left(\tau_{t}(y)\right),
$$

where [] denotes equivalence class and the limit exists for $\nu$-a.a. $y$ [33]. Now, it is shown in [15] that, if $y \in Y$ has dense orbit, then each $\lambda \in \operatorname{Resolvent}\left(L_{y}\right)$ gives rise to a continuous function $\phi_{\lambda}$ from $Y$ to the real projective space $P^{1}(\mathbf{R}) \cong K$. In fact, $\phi_{\lambda}$ arises in the following way. Begin with the relation $\operatorname{Resolvent}\left(L_{y}\right)=\{\lambda \in \mathbf{C} \mid$ equations $(5)_{y}$ have ED $\}$. Since the coefficient matrix in $(5)_{y}$ has trace zero, the projection $P=P_{y}: \mathbf{R}^{2} \rightarrow \mathbf{R}^{2}$ in the definition of ED must have one-dimensional range. Let $l_{y}$ be the range of $P_{y}$; then $l_{y}$ is a line through the origin in $\mathbf{R}^{2}$, i.e. an element of $\mathbf{P}^{1}(\mathbf{R})$. Define $\phi_{\lambda}: Y \rightarrow \mathbf{P}^{1}(\mathbf{R}): y \rightarrow l_{y}$. It turns out that $\alpha(\lambda)==_{\text {def }} h\left(\phi_{\lambda}\right) \in \mathbf{R}$ gives a labelling of the gaps: $\alpha(\lambda)$ depends only on the gap $I$ containing $\lambda$, and is 1-1 on the set of all gaps. We conclude that gaps can be labelled by the rotation number $[\mathbf{1 8}] \alpha(\lambda)$ for the random differential equations (2) $\omega$ when $\mu$ is ergodic.

These ideas can be used to "explain" geometrically a gap-labelling result of Bellissard and Scoppola [3], obtained using $C^{*}$-algebra techniques. They consider the (difference) operator equation

$$
L(x)_{n}=x_{n+1}+x_{n-1}+v(n) x_{n}=\lambda x_{n} \quad(n \in \mathbf{Z})
$$

where $v(n)=\tilde{v}\left(\theta_{0}+n\right)$ for a measurable but noncontinuous function $\tilde{v}$ on the circle $K=\{\theta \mid 0 \leq \theta<2 \pi\}$. As in [15], one can label the gaps for $L$ by using the Schwarzmann homomorphism on the suspension $\hat{V}$ of the hull $V$ of the function $v$.

2.7. Weyl $m$-functions and algebro-geometric potentials. Once again consider the Schrodinger equation

$$
L_{y} \phi=\left(\frac{-d^{2}}{d t^{2}}+y(t)\right) \phi=\lambda \phi,
$$

where $y \in Y \subset E_{p}$ is weakly compact and translation-invariant. Let $\nu$ be an ergodic measure on $Y$, and suppose that $\operatorname{Support}(\nu)=Y$. Let $\Phi_{ \pm}(t) \in L^{2}(0, \pm \infty)$ satisfy $L_{y} \phi=\lambda \phi$ for $\operatorname{Im} \lambda \neq 0$, and define the Weyl $m$-functions

$$
m_{ \pm}(y, \lambda)=\phi_{ \pm}^{\prime}(0) / \phi_{ \pm}(0) \quad(\operatorname{Im} \lambda \neq 0) .
$$

There is a relation between the behavior of $m_{ \pm}$as $\operatorname{Im} \lambda \rightarrow 0$ and the Lyapounov number $\beta=\beta(\lambda)$ of $(5)_{y}$ :

$$
\beta(\lambda)=\lim _{t \rightarrow \infty} \frac{1}{t} \ln \left\|\Phi_{y}(t)\right\|
$$

where $\Phi_{y}(t)$ is the fundamental matrix solution of $(5)_{y}$ satisfying $\Phi_{y}(0)=I$ (the limit exists and is independent of $y$ for $\mu$-a.a. $y$, by the Oseledec theorem). In fact, De Concini and Johnson [7] show that, if $\beta(\lambda)=0$ for a.a. $\lambda$ in an interval $I$, then $\lambda \rightarrow m_{+}(y, \lambda)$ extends holomorphically from $\{\lambda \mid \operatorname{Im} \lambda>0\}$ through $I$, and the extension equals $m_{-}(y, \lambda)$ for $\operatorname{Im} \lambda<0$. A similar change of sign occurs if one begins 
with $m_{-}(y, \lambda)$ in the upper half-plane. Using this result, the well-known algebrogeometric potentials $[\mathbf{2 7}, \mathbf{2 3}] y(t)$ are characterized in a simple and surprising way in the class of all pairs $(Y, \mu)$ : namely as those processes for which the spectrum $\Sigma$ of $L_{y}$ is a finite union of intervals for $\mu$-a.a. $y$, and for which $\beta(\lambda)=0$ a.e. in $\Sigma$.

By the construction presented above, we thus obtain an analogous characterization of the algebro-geometric potentials in the class of ergodic random Schrodinger operators $L_{\omega}$.

From the present discussion and from [7], one also obtains a characterization of the algebro-geometric AKNS potentials (named after [1]) in the class of ergodic random AKNS potentials.

3. Coppel's theorem. Our starting point in this section is a compact metric space $Y$ with continuous flow $\tau$, and a continuous cocycle (see 2.4) $\Phi: Y \times \mathbf{R} \rightarrow$ $\mathrm{GL}(n, \mathbf{C})$. For example, $Y$ might be a weakly compact subset of one of the spaces $E_{p}(p \geq 1)$ of $\S 2$, and $\Phi$ might be the cocycle defined by the corresponding family $(1)_{y}$ of differential equations.

Suppose that the cocyle $\Phi$ has exponential dichotomy (we abbreviate to ED). The definition is a natural generalization of that given in $\S 1$ : there are constants $K>0, \alpha>0$ and a continuous function $P: y \rightarrow P_{y}$ with domain $Y$ and range equal to the set of projections on $\mathbf{C}^{n}$ with the following properties:

(i) $\left\|\Phi_{y}(t) P_{y} \Phi_{y}^{-1}(s)\right\| \leq K e^{-\alpha(t-s)}(t \geq s ; y \in Y)$;

(ii) $\left\|\Phi_{y}(t)\left(I-P_{y}\right) \Phi_{y}^{-1}(s)\right\| \leq K e^{\alpha(t-s)}(t \leq s ; y \in Y)$.

It is often important to know that, if another cocycle $\Psi: Y \times \mathbf{R} \rightarrow \mathrm{GL}(n, \mathbf{C})$ is "close" to $\Phi$, then $\Psi$ also has ED, the corresponding projections are close to those of $\Phi$, and the projections vary with some degree of smoothness in $\Psi$. Both Coppel's result [6] and ours are of this type. As noted in the Introduction, the formulation of Sacker and Sell's roughness result [32] does not include a Lipschitz continuity statement.

3.1. THEOREM. Let $(Y, t)$ be a flow with $Y$ compact metric, i.e., $\tau: Y \times \mathbf{R} \rightarrow Y$ is continuous, $\tau(y, 0)=y$, and $\tau_{t} \circ \tau_{s}=\tau_{t+s}$ where $\tau_{t}(y) \equiv \tau(y, t)(y \in Y ; t, s \in \mathbf{R})$. Let $\Psi: Y \times \mathbf{R} \rightarrow \mathrm{GL}(n, \mathbf{C})$ be another cocyle, and let

$$
\delta=\sup _{y} \sup _{-1 \leq t \leq 1}\left\|\Phi_{y}(t)-\Psi_{y}(t)\right\| .
$$

Then there exists $\delta_{1}>0$ such that, if $\delta \leq \delta_{1}$, then $\Psi$ has ED with, say, family of projections $\left\{Q_{y} \mid y \in Y\right\}$; moreover, there exists a constant $K$ depending only on $\Phi$ such that $\sup _{y}\left\|P_{y}-Q_{y}\right\| \leq K \delta$.

The last part of Theorem 3.1 expresses Lipschitz continuity of $Q: y \rightarrow Q_{y}$ in the cocyle $\Psi$.

PROOF OF THEOREM 3.1. Our proof is motivated by arguments of Hirsch, Pugh, and Shub [14]. Let $B$ be the Banach space of all bounded (not necessarily continuous) maps $b: Y \rightarrow \mathbf{C}^{n}$ with norm $\|b\|_{\infty}=\sup _{y}|b(y)|$. Here $|\cdot|$ is the Euclidean norm on $\mathbf{C}^{n}$. Define a one-parameter group of bounded linear transformations

$$
T_{\Phi}(t): B \rightarrow B: T_{\Phi}(t) \cdot b(y)=\Phi\left(\tau_{-t}(y), t\right) \cdot b\left(\tau_{-t}(y)\right) .
$$

Define $\tilde{P}: B \rightarrow B$ by $(\tilde{P} b)(y)=P_{y}(b(y))$ for $b \in B$. Then $\tilde{P}$ is a continuous projection on $B$, and it follows from ED that $\tilde{P}$ commutes with $T_{\Phi}(t)$ for all $t \in \mathbf{R}$. 
By ED and the spectral radius formula [30], the spectrum of $T_{\Phi}(t)$ restricted to $\operatorname{Im} \tilde{P}$ is contained in the open disc with radius $e^{-\alpha t / 2}$. Similarly, the spectrum of $T_{\Phi}(-t)=T_{\Phi}(t)^{-1}$ restricted to Ker $\tilde{P}$ is contained in this disc. Therefore the unit circle $C$ separates the spectrum of $T_{\Phi}(1)$ into two disjoint closed sets, one inside $C$ and one outside $C$.

Next let $C$ be positively oriented. Define a projection $P_{*}: B \rightarrow B$ by

$$
P_{*}=\frac{1}{2 \pi i} \int_{C}\left(\lambda-T_{\Phi}(1)\right)^{-1} d \lambda .
$$

By [30, Chapter 12] and continuity of $\Phi_{y}(t)$, the image of $P_{*}$ equals $\{b \in B \mid$ $\left.\lim _{t \rightarrow \infty}\left\|T_{\Phi}(t) \cdot b\right\|_{\infty}=0\right\}$, and the kernel of $P_{*}$ equals $\left\{b \in B \mid \lim _{t \rightarrow \infty}\left\|T_{\Phi}(-t) \cdot b\right\|_{\infty}\right.$ $=0\}$.

Define $T_{\Psi}(t)$ to be the one-parameter group of bounded linear transformations on $B$ defined by the cocycle $\Psi$. We can choose $\delta_{1}>0$ (depending only on $\Phi$ ) such that, if $\left\|T_{\Psi}(1)-T_{\Phi}(1)\right\|<\delta_{1}$, then the spectrum of $T_{\Psi}(1)$ is also separated into two closed disjoint subsets by $C$. Let

$$
Q_{*}=\frac{1}{2 \pi i} \int_{C}\left(\lambda-T_{\Psi}(1)\right)^{-1} d \lambda,
$$

and let $M=\|\left(\lambda-T_{\Phi}(1)^{-1} \|_{\infty}\right.$, where the norm is of course the operator norm induced by $\|\cdot\|_{\infty}$ on $B$. Then

$$
\left\|Q_{*}-P_{*}\right\|_{\infty} \leq M \sum_{n=1}^{\infty} M^{n} \cdot\left\|T_{\Psi}(1)-T_{\Phi}(1)\right\|_{\infty}^{n} .
$$

Choosing, for example, $\delta_{1} \leq 1 / 2 M$, and $K=2 M^{2}$, we obtain with $\delta=$ $\left\|T_{\Phi}(1)-T_{\Psi}(1)\right\|_{\infty}$ :

$$
\left\|Q_{*}-P_{*}\right\|_{\infty} \leq K \delta .
$$

Now we show that $\left(Q_{*} b\right)(y)=Q_{y}(b(y))$ where $Q_{y}: \mathbf{C}^{n} \rightarrow \mathbf{C}^{n}$ is a projection $(y \in Y)$. To do so, note first that (perhaps shrinking $\delta_{1}$ if necessary) we can assume that there is a bounded linear map $h$ : Range $P_{*} \rightarrow \operatorname{Ker} P_{*}$ such that Range $Q_{*}=$ $\left\{b+h(b) \mid b \in\right.$ Range $\left.P_{*}\right\}$. In fact, $h(b)$ can be characterized as the unique element of $\operatorname{Ker} P_{*}$ such that $\left\|T_{\Psi}(t)(b+h(b))\right\|_{\infty} \rightarrow 0$ as $t \rightarrow+\infty[30]$.

This being said, let $y \in Y, v \in \operatorname{Range} P_{y}$, and set

$$
b_{v}(\tilde{y})= \begin{cases}v, & \tilde{y}=y \\ 0, & \tilde{y} \neq y\end{cases}
$$

Define $h_{y}$ : Range $P_{y} \rightarrow \operatorname{Ker} P_{y}$ by $h_{y}(v)=h\left(b_{v}\right)(y)$. Let $b \in$ Range $P_{*}$. Using the uniqueness of $h(b)$, the definition of $T_{\Psi}(t)$, and the characterization Range $Q_{*}=$ $\left\{c \in B \mid \lim _{t \rightarrow \infty}\left\|T_{\Psi}(t) \cdot c\right\|_{\infty}=0\right\}$, we see that $h(b)(y)=h_{y}(b(y))(y \in Y)$.

Shrinking $\delta_{1}$ again, we can find a bounded linear map $p$ : Ker $P_{*} \rightarrow$ Range $P_{*}$ such that $\operatorname{Ker} Q_{*}=\left\{b+p(b) \mid b \in \operatorname{Ker} P_{*}\right\}$. Reasoning much as we did just above, we can write $p(b)(y)=p_{y}(b(y))$. It now follows that $\left(Q_{*} b\right)(y)=Q_{y}(b(y))$ where $Q_{y}$ is a projection $(b \in B, y \in Y)$. It follows from this statement and [30, Chapter 12] that (i) and (ii) hold with $\Psi_{y}, Q_{y}$ in place of $\Phi_{y}, P_{y}$.

It must still be shown that $y \rightarrow Q_{y}$ is continuous. First of all, write $Y=$ $Y_{0} \cup Y_{1} \cup \cdots \cup Y_{n}$ where $Y_{i}=\left\{y \in Y \mid \operatorname{dim}\right.$ range $\left.P_{y}=i\right\}$. It follows from continuity of $y \rightarrow P_{y}$ and (i), (ii) that each $Y_{i}$ is an open and closed, invariant subset of $Y$. 
Shrinking $\delta_{1}$ and using $\left\|Q_{*}-P_{*}\right\|_{\infty} \leq K \delta_{1}$, we can assume $\operatorname{dim} Q_{y}=\operatorname{dim} P_{y}(y \in$ $Y)$. Next note that, if $\sup _{t}\left\|\Psi_{y}(t) x\right\|<\infty$ for some $y \in Y$ and $x \in \mathbf{C}^{n}$, then $x=0$. Since $\operatorname{dim} Q_{y}$ is constant on each minimal subset of $Y_{i}$, a result of Sacker and Sell [32] implies that $\Psi$ has ED on $Y_{i}(0 \leq i \leq n)$. Thus $y \rightarrow Q_{y}$ is continuous on each $Y_{i}$, hence on all of $Y$.

Let us now show how Theorem 3.1 can be applied to systems like those considered in $\S 2$. For $p \geq 1$, let $Y \subset E_{p}$ be a weakly compact invariant set. Let $\Phi_{y}$ be the cocycle defined by equations $(1)_{y}$. Let $V$ be a weakly compact, invariant subset of $E_{r}$ for $r \geq 1$. Consider the product space $Y \times X$ with the product flow $\hat{\tau}$ : thus $\hat{\tau}((y, v), t)=\left(\tau_{t}(y), \tau_{t}(v)\right)$. Define $\eta_{1}: Y \times V \rightarrow Y:(y, v) \rightarrow y$ and $\eta_{2}: Y \times$ $V \rightarrow E_{\min (r, p)}:(y, v) \rightarrow y+v$. Then $\eta_{1}$ and $\eta_{2}$ are flow-preserving maps. If $z=(y, v) \in Y \times V$, define $\Phi_{z}(t)=\Phi\left(\eta_{1}(z), t\right)$ and $\Psi_{z}(t)=\Gamma(t)$, where $\Gamma(t)$ is the fundamental matrix solution of $x^{\prime}=(y(t)+v(t)) x$ satisfying $\Gamma(0)=I$. Then $\Phi$ and $\Psi$ are continuous cocyles on $Y \times V$ with values in $\operatorname{GL}(n, \mathbf{C})$.

Given $E_{p}$ the norm $\|\cdot\|_{p}$ defined by $\|w\|_{\infty}=\sup _{t}\|w(t)\|$ if $p=\infty$,

$$
\|w\|_{p}^{p}=\sup _{t} \int_{t}^{t+1}\|w(s)\|^{p} d s \quad \text { for } 1 \leq p<\infty .
$$

One checks using Gronwall's inequality that

$$
\sup _{-1 \leq t \leq 1}\left\|\Phi_{z}(t)-\Psi_{z}(t)\right\| \leq M\|v\|_{r}, \quad z=(v, y)
$$

where $M$ is a constant depending only on the cocycle $\Phi$. Thus if $\sup \left\{\|v\|_{r} \mid v \in V\right\}$ is sufficiently small, then Theorem 3.1 may be applied: if $\Phi$ has ED, then so does $\Psi$, and the projections are Lipschitz in $v$ with respect to the norm $\|\cdot\|_{r}$.

We finish the section by considering a frequently-encountered situation. Consider

$$
x^{\prime}=A(t) x \quad\left(x \in \mathbf{C}^{n}\right),
$$

where $A$ is uniformly locally $L^{p}(p \geq 1)$. Suppose that $Y=\operatorname{cls}\left\{\tau_{t}(A) \mid t \in \mathbf{R}\right\}$ is compact in $E_{p}$ (this is automatic if $p>1$; see $\S 2$ ). Suppose that (3) has ED with projection $P$. Then so does the set of equations $(6)_{y}$, where $y$ runs over $Y$. Let $B(t)$ be a function which is uniformly locally $L^{r}$, and such that $V=\operatorname{cls}\left\{\tau_{t}(B) \mid t \in \mathbf{R}\right\}$ is compact in $E_{r}$. Suppose that $\sup \left\{\|v\|_{r} \mid v \in V\right\}=\sup \left\{\left\|\tau_{t}(B)\right\|_{r} \mid t \in \mathbf{R}\right\}<\varepsilon$, where $\varepsilon$ is chosen so that $M_{\varepsilon}$ is less than the $\delta_{1}$ in Theorem 3.1. Then the perturbed equation

$$
x^{\prime}=(A(t)+B(t)) x
$$

has $\mathrm{ED}$, and the corresponding projection $Q$ satisfies

$$
\|Q-P\| \leq K \cdot \sup _{t}\left\|\tau_{t}(B)\right\|_{r}
$$

where $K$ depends only on equation (6).

4. The almost-periodic Schrodinger equation. Let $q(t)$ be a real-valued, Bohr almost-periodic function of $t$, and consider the Schrodinger operator $L=$ $-d^{2} / d t^{2}+q(t)$ with potential $q$. The corresponding Schrodinger equation is then

$$
L \phi=\lambda \phi \quad(\lambda \in \mathbf{C})
$$

or in system form

$$
x^{\prime}=\left(\begin{array}{c}
\phi \\
\phi^{\prime}
\end{array}\right)^{\prime}=\left(\begin{array}{cc}
0 & 1 \\
-\lambda+q(t) & 0
\end{array}\right) x
$$


The spectrum $\Sigma$ of $L$ (as a selfadjoint operator on $L^{2}(\mathbf{R})$ ) turns out to be a nontrivial object to study. One expects that, for generic $q, \Sigma$ is a Cantor-like subset of $\mathbf{R}$ (i.e., closed and nowhere dense). Our purpose here is to prove a partial result in this direction.

To formulate it, let $Y=\operatorname{cls}\left\{\tau_{t}(q) \mid t \in \mathbf{R}\right\}$ be the hull of $q$. Unlike the previous sections, the closure here is taken with respect to the topology of uniform convergence on all of $\mathbf{R}$. Then $Y$ is compact, and translation $\tau$ defines a continuous flow on $Y$. For fixed $\lambda \in \mathbf{C}$, we obtain a family of equations

$$
x^{\prime}=\left(\begin{array}{cc}
0 & 1 \\
-\lambda+y(t) & 0
\end{array}\right) x \quad(y \in Y) .
$$

It is known [10] that $Y$ can be given the structure of a compact, abelian topological group in such a way that $q$ is the identity and (* denotes group multiplication) $\left(\tau_{t} q\right) *\left(\tau_{s} q\right)=\tau_{t+s} q(t, s \in \mathbf{R})$. Let $\mu$ be normalized Haar measure on $Y$.

Now we introduce the Lyapounov exponent $\beta(\lambda)$ of equations $(5)_{y}$. For fixed $\lambda \in \mathbf{C}$, let $\Phi_{y}(t)$ be the fundamental matrix solution of $(5)_{y}$ such that $\Phi_{y}(0)=I$. Define

$$
\beta(\lambda)=\lim _{t \rightarrow \infty} \frac{1}{t} \ln \left\|\Phi_{y}(t)\right\| ;
$$

the Oseledec theorem [28] implies that the limit exists and is independent of $y$ for $\mu$-a.a. $y$.

It is known that $\lambda$ is in the resolvent of $L_{y}=-d^{2} / d t^{2}+y(t)$ if and only if equations (5) $)_{y}$ have $\operatorname{ED~[17].~It~is~also~known~that,~if~} \beta(\lambda)>0$, and if equations $(5)_{y}$ do not have $\mathrm{ED}$, then the solutions display quite complicated behavior. In fact, it was not known until Millionscikov's example [35] of 1969 that an almost periodic ordinary differential equation without ED whose coefficient matrix has trace zero can have strictly positive Lyapounov exponent (see also Vinograd [36]).

We will show that, for generic $q$, one of the following holds.

(i) The spectrum $\Sigma$ of $L=-d^{2} / d t^{2}+q(t)$ is a Cantor set.

(ii) If $I \subset \Sigma$ is an interval, then $\beta(\lambda)>0$ for $\lambda$ in a subset of $I$ of positive Lebesgue measure; thus equation $(5)_{y}$ has the property that $\left\|\Phi_{y}(t)\right\|$ grows exponentially for $\mu$-a.a. $y$.

We formulate our theorem so as to make clear that smoothness assumptions on $q$ and Diophantine conditions do not play a role. Thus suppose

$$
q(t)=Q\left(\alpha_{1} t, \ldots, \alpha_{s} t\right) \quad(t \in \mathbf{R}),
$$

where $Q\left(\theta_{1}, \ldots, \theta_{s}\right)$ is $2 \pi$-periodic in each of the variables $\theta_{1}, \ldots, \theta_{s}(s \geq 2)$. Suppose moreover that $\alpha_{1}, \ldots, \alpha_{s}$ are independent over the rational numbers $\mathbf{Q}$. Let $r>0$, and introduce the set $D_{r}$ of multiply periodic functions $Q$ which are analytic and uniformly bounded in the complex strip $\left|\operatorname{Im} \theta_{i}\right|<r(1 \leq i \leq s)$, with period $2 \pi$ in each complex variable $\theta_{1}, \ldots, \theta_{s}$. Then $D_{r}$ is a Banach space in the sup-norm $\|\cdot\|_{\infty}$.

4.1. Proposition. For $Q \in D_{r}$, write $q(t)=Q\left(\alpha_{1} t, \ldots, \alpha_{s} t\right)$. There is a residual subset of $Q \in D_{r}$ with the property that, if $I \subset \mathbf{R}$ is an interval in the spectrum $\Sigma=\Sigma_{Q}$ of $L=-d^{2} / d t^{2}+q(t)$, then $\beta(\lambda)>0$ on a subset of $I$ of positive Lebesgue measure. 
PROOF. Let $\left\{I_{k} \mid k \geq 1\right\}$ be an enumeration of the open bounded subintervals of $\mathbf{R}$ with rational endpoints. Let $F_{k}=\left\{Q \in D_{r} \mid \beta(\lambda) \equiv \beta_{Q}(\lambda)=0\right.$ for a.a. $\left.\lambda \in I_{k}\right\}$.

We first show that $F_{k}$ is closed in $D_{r}$ for each $k$. To do so, we appeal to the theory of the Floquet exponent $[\mathbf{1 8}, \mathbf{2 0}, \mathbf{7}]$. Briefly, there is a holomorphic function $w(\lambda) \equiv w_{Q}(\lambda)$, defined for $\operatorname{Im}>0$, having positive real part there (and also positive imaginary part), such that, for all $\lambda \in \mathbf{R}, \lim _{\varepsilon \rightarrow 0^{+}} \operatorname{Re} w(\lambda+i \varepsilon)=\beta(\lambda)$. The function $w$ is uniformly bounded on each set of the form $\{\lambda|\operatorname{Im} \lambda>0,| \lambda \mid<R\}$, and the bound depends only on $R$ and on $\|Q\|_{\infty}$.

Now let $Q_{l} \rightarrow Q$ in $D_{r}$, and write $\beta_{l}=\beta_{Q_{l}}, w_{l}=w_{Q_{l}}$. Suppose each $Q_{l}$ is in $F_{k}$. Then each $w_{l}$ extends holomorphically to the disc $J_{k}$ with diameter $I_{k}$ : this is because $i w_{l}$ can be Schwarz-reflected through $I_{k}$. Writing $\tilde{w}_{l}$ for the extension, we have $\operatorname{Re} \tilde{w}_{l}(\lambda)=0$ for $\lambda \in I_{k}$. Moreover, using the previous paragraph, $\left\{\tilde{w}_{l} \mid l \geq 1\right\}$ is uniformly bounded on $J_{k}$. Since one can show without difficulty that $w_{l}(\lambda) \rightarrow$ $w_{Q}(\lambda)$ for $\operatorname{Im} \lambda>0$ (e.g., $[20]$ ), the $\operatorname{limit}_{l \rightarrow \infty} \tilde{\lim }_{l}(\lambda) \equiv \tilde{w}(\lambda)$ exists for each $\lambda \in J_{k}$, is holomorphic on $J_{k}$, and $\tilde{w}(\lambda)=w_{Q}(\lambda)$ for $\operatorname{Im} \lambda>0$. Hence $\beta_{Q}(\lambda)=0$ for all $\lambda \in I_{k}$. It follows that $Q \in F_{k}$; i.e., $F_{k}$ is closed, as we wanted to show.

Next we show that $F_{k}$ is nowhere dense. We use results of Johnson and Moser [18], Moser and Poschl [26], and De Concini and Johnson [7]. Fix $Q \in F_{k}$, and let $Y$ be the hull of $q(t)$. Then $Y$ may be identified with a quotient of the $s$-torus defined by the angular variables $\theta_{i}, 0 \leq \theta_{i} \leq 2 \pi, 1 \leq i \leq s$. In fact, to each $y \in Y$ there corresponds $\left(\theta_{1}^{0}, \ldots, \theta_{s}^{0}\right)$ such that

$$
y(t)=Q\left(\theta_{1}^{0}+\alpha_{1} t, \ldots, \theta_{s}^{0}+\alpha_{s} t\right) .
$$

Let $m_{ \pm}(\lambda)=m_{ \pm}(y, \lambda)$ be the Weyl $m$-functions corresponding to $y \in Y(\operatorname{Im} \lambda \neq$ $0)$. These functions are described in e.g., [5, Chapter 9]; for example, $m_{+}(\lambda)=$ $\phi^{\prime}(0) / \phi(0)$ where $0 \neq \phi$ satisfies $\left(-d^{2} / d t^{2}+y(t)\right) \phi=\lambda \phi$ and $\phi \in L^{2}(0, \infty)$. Then $m_{ \pm}(\lambda)$ are holomorphic for $\operatorname{Im} \lambda \neq 0$, and $\operatorname{sgn}\left[\operatorname{Im} m_{ \pm}(\lambda) \cdot \operatorname{Im} \lambda\right]= \pm 1$. According to [7], the condition $\beta_{Q}(\lambda)=0$ a.e. on $I_{k}$ implies that $\lambda \rightarrow m_{+}(y, \lambda)$ extends holomorphically from $\{\operatorname{Im} \lambda>0\}$ through $I_{k}$, and the extension $\tilde{m}_{+}(y, \lambda)$ equals $m_{-}(y, \lambda)$ for $\operatorname{Im} \lambda<0(y \in Y)$. Moreover the extension is jointly continuous in $Y \times\left\{\lambda \mid \operatorname{Im} \lambda \neq 0\right.$ or $\left.\lambda \in I_{k}\right\}$, and $\operatorname{Im} \tilde{m}_{+}(y, \lambda)>0$ for $\lambda \in I_{k}$. Analogous statements hold for $\lambda \rightarrow m_{-}(y, \lambda)$; with the corresponding notation, $\operatorname{Im} \tilde{m}_{-}(y, \lambda)<0$ for $\lambda \in I_{k}$.

Define $\phi_{ \pm}(t)=\exp \int_{0}^{t} m_{ \pm}\left(\tau_{s} q, \lambda\right) d s$ for $\lambda \in I_{k}$. Then the vectors $\left(\phi_{ \pm}(t), \phi_{ \pm}^{\prime}(t)\right)^{t}$ satisfy equation $(5)_{q}$ (i.e., $y=q$ in equation $(5)_{y}$ ). Moreover the complex lines $l_{ \pm}(t)$ determined by these solutions are bounded away from each other on the projective space $\mathbf{P}^{1}(\mathbf{C})$ for all $t \in \mathbf{R}$ (precisely because $\operatorname{Im} \tilde{m}_{+}\left(\tau_{s} q, \lambda\right)>0$ and $\operatorname{Im} \tilde{m}_{-}\left(\tau_{s} q, \lambda\right)<0$ for $\left.\lambda \in I_{k}(s \in \mathbf{R})\right)$. Still more, if $\left(\psi(t), \psi^{\prime}(t)\right)$ is any nonzero real solution of $(5)_{q}$, then the complex line that it determines is bounded away from both $l_{+}(t)$ and $l_{-}(t)$ for all $t \in \mathbf{R}$. If $\Phi_{q}(t)$ is the fundamental matrix solution of $(5)_{q}$ satisfying $\Phi_{q}(0)=I$, then $\operatorname{det} \Phi_{q}(t) \equiv 1$ by Liouville's formula; it follows easily that all solutions of $(5)_{q}$ are bounded and (if nonzero) bounded away from zero. Hence $\phi_{ \pm}(t)$ are bounded.

Since $s \rightarrow m_{ \pm}\left(\tau_{s} q, \lambda\right)$ are almost periodic, a theorem of H. Bohr (e.g., [12, Chapter 6]) implies that $\phi_{ \pm}(t)$ are almost periodic. Hence all solutions of $(5)_{q}$ are almost periodic. 
It is time to introduce the rotation number $\alpha(\lambda)=-\lim _{\varepsilon \rightarrow 0^{+}} \operatorname{Im} w(\lambda+i \varepsilon)$ for $\lambda \in \mathbf{R}[\mathbf{1 8}, \mathbf{1 3}]$. In [18], it is shown that $-\alpha$ is continuous, monotone nondecreasing, and

$$
\alpha(\lambda)=\lim _{t \rightarrow \infty} \frac{1}{t} \operatorname{Arg}\left(\psi(t)+i \psi^{\prime}(t)\right)
$$

for each real solution $\left(\psi(t), \psi^{\prime}(t)\right)^{t}$ of $(5)_{q}$. One can show that

$$
\alpha(\lambda)=\lim _{t \rightarrow \infty} \frac{1}{t} \operatorname{Arg} \phi_{ \pm}(t)
$$

see [18] for a proof. Hence

$$
\phi_{+}(t)=\left|\phi_{+}(t)\right| \exp (i \alpha(\lambda) t+\gamma(t))
$$

where $\gamma(t)$ is almost periodic with mean value zero and frequency module [12] contained in the frequency module $M$ of $\phi_{+}(t)$. These last statements follow from the theorem of Bohr used earlier. We remark that $M=$ frequency module of $q=\left\{\sum_{i=1}^{s} n_{i} \alpha_{i} \mid n_{i}=0, \pm 1, \ldots\right\}$.

Next, $I_{k}$ is in the spectrum of $L$ because $\beta=0$ on $I_{k}$ (e.g., [20]). By [18], $-\alpha$ increases exactly on the spectrum of $L$. Thus there exists $\lambda_{0} \in I_{k}$ such that $2 \alpha\left(\lambda_{0}\right)$ is in $M$.

Using this fact and $(*)$, we see that Lemma 6.1 of Moser and Poschl [26] can be applied to $(5)_{q}$ when $\lambda=\lambda_{0}$. Thus there is a trigonometric polynomial $P\left(\theta_{1}, \ldots, \theta_{s}\right)$ such that, if $\tilde{q}(t)=Q\left(\alpha_{1} t, \ldots, \alpha_{s} t\right)+\varepsilon P\left(\alpha_{1} t, \ldots, \alpha_{s} t\right)$ and $\varepsilon$ is small, then the resolvent of $\hat{L}=-d^{2} / d t^{2}+\tilde{q}(t)$ intersects $I_{k}$. Thus $Q+\varepsilon P$ is not in $F_{k}$; i.e. $F_{k}$ has no interior points.

Finally, let $F_{0}=\bigcup_{k=1}^{\infty} F_{k}$. Then $D_{r} \backslash F_{0}$ is a residual subset of $D_{r}$ such that, if $Q \in D_{r} \backslash F_{0}$, then either $\Sigma_{Q}$ is a Cantor set, or if $I \subset \Sigma_{Q}$ is an interval, then $\beta_{Q}(\lambda)>0$ on a subset of $I$ of positive Lebesgue measure. This completes the proof of 4.1 .

4.2. REMARKS. (i) Of course the argument used above applies to many other Banach spaces of almost periodic functions.

(ii) Using [ 7 and 13], one can prove the analogue of 4.1 for the AKNS [1] operator

$$
L=\left(\begin{array}{cc}
0 & -1 \\
1 & 0
\end{array}\right)\left[\frac{d}{d t}-A(t)\right]
$$

for almost periodic matrices $A(t)$ of trace zero.

\section{REFERENCES}

1. M. Ablowitz, D. Kaup, A. Newell, and H. Segur, The inverse scattering transform: Fourier analysis for non-linear systems, Stud. Appl. Math. 53 (1974), 249-315.

2. J. Avron and B. Simon, Almost periodic Schrodinger operators. II, The integrated density of states, Duke Math. J. 50 (1983), 369-391.

3. J. Bellissard and E. Scoppola, The density of states for almost-periodic Schrodinger operators and the frequency module: a counterexample, Comm. Math. Phys. 85 (1982), 301-308.

4. J. Bellissard and B. Simon, Cantor spectrum for the almost Mathieu equation, J. Funct. Anal. 48 (1982), 408-419. 
5. E. Coddington and N. Levinson, Theory of ordinary differential equations, McGraw-Hill, New York, 1955.

6. A. Coppel, Dichotomies in stability theory, Lecture Notes in Math., vol. 629, Springer-Verlag, Berlin and New York, 1978.

7. C. De Concini and R. Johnson, The algebraic-geometric AKNS potentials, Ergodic Theory Dynamical Systems (to appear).

8. I. Dinaburg and Ya. Sinai, On the one-dimensional Schrodinger equation with quasi-periodic potential, Functional Anal. Appl 9 (1975), 8-21.

9. J. Doob, Stochastic processes, Wiley, New York, 1953.

10. R. Ellis, Lectures on topological dynamics, Benjamin, New York, 1967.

11. R. Ellis and R. Johnson, Topological dynamics and linear differential systems, J. Differential Equations 44 (1982), 21-39.

12. A. Fink, Almost periodic differential equations, Lecture Notes in Math., Vol. 377, SpringerVerlag, Berlin and New York, 1974.

13. R. Giachetti and R. Johnson, Spectral theory of two-dimensional, almost-periodic differential operators and its relation to certain non-linear evolution equations, Nuovo Cimento B (11) 82 (1984), 125-168.

14. M. Hirsch, C. Pugh, and M. Shub, Invariant manifolds, Lecture Notes in Math., Vol. 583, Springer-Verlag, Berlin and New York, 1977.

15. R. Johnson, Exponential dichotomy, rotation number, and linear differential equations with bounded coefficients, J. Differential Equations 61 (1986), 54-78.

16. __ m-functions and Floquet exponents for linear differential systems, Ann. Mat. Pura Appl. (to appear).

17. _ The recurrent Hill's equation, J. Differential Equations 46 (1982), 165-194.

18. R. Johnson and J. Moser, The rotation number for almost periodic potentials, Comm. Math. Phys. 84 (1982), 403-438.

19. R. Johnson, K. Palmer and G. Sell, Ergodic properties of linear dynamical systems, SIAM J. Math. Anal. (to appear).

20. S. Kotani, Lyapounov indices determine absolutely continuous spectra of stationary random onedimensional Schrodinger operators, Proc. Taniguchi Sympos. SA, Katata, 1982, pp. 225-247.

21. __, On an inverse problem for random Schrodinger operators, Particle Systems, Random Media and Large Derivations, edited by Richard Durrett, Contemporary Math., vol. 41, Amer. Math. Soc., Providence, R.I., 1985, pp. 267-280.

22. __ Support theorems for random Schrodinger operators, Comm. Math. Phys. 97 (1985), 443-452.

23. H. McKean and P. van Moerbeke, The spectrum of Hill's equation, Invent Math. 30 (1975), 217-274.

24. R. Miller and G. Sell, Volterra integral equations and topological dynamics, Mem. Amer. Math. Soc., No. 102 (1970).

25. V. Millionscikov, Proof of the existence of irregular systems of linear differential equations 2 ith almost periodic coefficients, J. Differential Equations 4 (1968), 203-205.

26. J. Moser and J. Poschl, An extension of a result by Dinaburg and Sinai on quasi-periodic potentials, Comment. Math. Helv. 59 (1984), 39-85.

27. S. Novikov, The periodic Korteweg-de Vries problem, Functional Anal. Appl. 8 (1974), 54-66.

28. V. Oseledec, A multiplicative ergodic theorem. Lyapounov characteristic numbers for dynamical systems, Trans. Moscow Math. Soc. 19 (1968), 197-231.

29. K. Palmer, On Cherry's example of a differential equation with a homoclinic point, preprint, 1985.

30. F. Riesz and B. Sz.-Nagy, Vorlesungen uber Funktionalanalysis, Deutscher Verlag der Wissenschaften, Berlin, 1973.

31. R. Sacker and G. Sell, Existence of dichotomies and invariant splittings for linear differential systems. I, J. Differential Equations 15 (1974), 429-458.

32. _ , A spectral theory for linear differential systems, J. Differential Equations 27 (1978), 320-358. 
33. S. Schwarzmann, Asymptotic cycles, Ann. of Math. 66 (1957), 270-284.

34. J. Selgrade, Isolated invariant sets for flows on vector bundles, Trans. Amer. Math. Soc. 203 (1975), 359-390.

35. G. Sell, Topological dynamics and linear differential equations, Van Nostrand-Reinhold, New York, 1971.

36. R. Vinograd, A problem suggested by N. P. Erugin, J. Differential Equations 11 (1975), 474478.

Department of Mathematics, Universitat Heidelberg, SFB 123, D-6900 HeiDELBERG, FEDERAL REPUBLIC OF GERMANY

Current address: Department of Mathematics, University of Southern California, Los Angeles, California 90089 